\title{
Interaction between Locomotion and Self-Care for Patients with Stroke in Severity Classification Based on the Motor Functional Independence Measure upon Admission to the Recovery Ward
}

\author{
Takashi Kimura1,2 \\ ${ }^{1}$ Department of Physical Therapy, ASO Rehabilitation College, Higashi-Hie, Hakata-ku, Fukuoka-Shi, Fukuoka, Japan \\ ${ }^{2}$ Department of Rehabilitation Medicine, Saga University Hospital, Nabeshima, Saga-shi, Saga, Japan \\ Email: t.kimufuku@gmail.com
}

How to cite this paper: Kimura, T. (2020) Interaction between Locomotion and Self-Care for Patients with Stroke in Severity Classification Based on the Motor Functional Independence Measure upon Admission to the Recovery Ward. Open Journal of Therapy and Rehabilitation, 8, 164-182. https://doi.org/10.4236/ojtr.2020.84015

Received: October 3, 2020

Accepted: November 6, 2020

Published: November 9, 2020

Copyright $\odot 2020$ by author(s) and Scientific Research Publishing Inc. This work is licensed under the Creative Commons Attribution International License (CC BY 4.0). http://creativecommons.org/licenses/by/4.0/

\section{Open Access}

\begin{abstract}
Background: A correlation between self-care and gait in stroke patients has been shown. However, there are few reports suggesting an interaction between self-care and gait. Purpose: To investigate the interaction between locomotion and improvements in self-care. Participants and Methods: We retrospectively analyzed 3034 stroke patients who were registered in the Japanese Rehabilitation Database. Using their data, patients were classified into three groups (severe, moderate, slight) based on the motor functional independence measure upon admission, and data were modified as mean-centered values. We performed a correlation analysis to evaluate the relationship among all the collected data. Subsequently, a hierarchical multiple regression analysis was performed to evaluate interaction using the self-care motor score from the Functional Independent Measure (FIM) as the dependent variable. Model 1 used two independent variables (National Institutes of Health Stroke Scale and cognitive FIM score), model 2 used two independent variables (locomotion gain and gain of an item with the strongest correlation coefficient to the de-pendent variable), and model 3 used a mean-centering value, which was added to model 2 . The simple slope was used for further analysis. Results: Locomotion showed an interaction with self-care, except in the slight group. The $\mathrm{R}^{2}$ changes in models 1 and 2 were significant in the following: grooming, dressing lower body, and bladder management in the severe group ( $\mathrm{p}<0.01$ ), and only dressing upper body in the moderate group $(\mathrm{p}<0.01)$. Results of the simple slope analysis were significant for grooming, bowel management, and dressing lower body in the severe group and for dressing
\end{abstract}


upper body in the moderate group. Conclusion: Locomotion demonstrated interaction with improving self-care, mainly in the severe group. Therefore, for moderate and slight cases, an intervention that not only involves locomotion training but also focuses on improving activities of daily living should be considered.

\section{Keywords}

Interaction, Stroke, Gait, Self-Care, Recovery Rehabilitation

\section{Introduction}

Gait disturbance is one of the most common consequences of stroke. Patients with stroke have particular functional disabilities that may cause locomotion reduction and, subsequently, affect their activities of daily living (ADL) [1] [2] [3]. Previous studies have reported that the recovery progress appears mainly within 3 months and moderately until 6 months post-stroke. Early and intensive rehabilitation can improve ADL for inpatients, and intensive intervention in the recovery phase reportedly improves ADL. Many factors affect improvement in $\mathrm{ADL}$, and there is a significant correlation between locomotion and ADL, especially during the acute phase in stroke; locomotion improvement may positively affect ADL performance [1] [4] [5]. Moreover, ADL prognostic prediction is an important factor in considering discharge to the patient's home. Thus, in the early stages of stroke rehabilitation, interventions focus on achieving locomotion. Additionally, it has been reported that gait training exercises influence ADL performance in the early post-stroke period [1] [2] [3] [5]. Therefore, locomotion was addressed early as the main intervention in stroke rehabilitation despite difficulties in ADL performance; however, these reports focused on the association between motor Functional Independence Measure (m-FIM) and $\mathrm{ADL}$ as a correlation and not from the interaction viewpoint.

Previous studies have shown that many factors, including gait and ADL, are correlated with locomotion [6] [7] [8]. These results have shown that patients with stroke who have a higher locomotion ability may have higher m-FIM scores and could probably perform ADL better, thus, preventing ADL decline. Recently, it has been shown that the trunk function has a stronger relationship with improving ADL than the hemiplegic side function [9], and it influences motor and balance functions (e.g., sitting and standing balance) [3] [4] [10]. Improving $\mathrm{ADL}$ and gait were influenced by the load and frequency of training [11]. Furthermore, an improvement was observed by increasing gait training time 1.5 times the baseline; however, the effect after 1 year was not significant [12] [13]. Additionally, the affected upper limb is a predictor for outcomes of ADL 3 months post-stroke in ADL improvement. There have been many reports about the correlation between ADL and gait post-stroke. Research from an interaction viewpoint showed a significant interaction between ADL performance and de- 
pressive symptoms of chronic stroke diseases [14]. Interaction is defined as an impact that occurs when two or more factors affect each other. Previous reports have shown that the affected upper limbs and balance function have an interaction with $\mathrm{ADL}$, and it has been revealed that cognition and gait could interact through a cognitive-motor interference [15] [16]. In paralysis after stroke, there was a significant interaction effect between gait factors and the affected limb. However, only a few reports have been published about an interaction between ADL and gait, that is to say, a two-way effect [17].

Despite the large amount of literature available on the relationship between locomotion and $\mathrm{ADL}$, the interaction between increased locomotion performance and improvements in individual ADL has not been sufficiently elucidated in patients with stroke. Intervention related to ADL could differ depending on the existence of an interactive relationship between ADL and locomotion. Thus, considering the interaction between gait and ADL would lead to a more significant improvement in ADL. This could be beneficial for improving ADL performance through gait training during the rehabilitation of patients with stroke.

Therefore, it is important to clarify the existence of an interaction between locomotion and improved ADL performance.

This study aimed to investigate the interaction between locomotion and improvement in self-care activities based on the m-FIM score.

\section{Materials and Method}

\subsection{Subject}

The medical data of 6,875 patients with stroke were extracted from the Japan Rehabilitation Database (JRD) in the stroke/recovery rehabilitation phase ward (January 2016 version, https://square.umin.ac.jp/JARD/). We used anonymized observational data obtained in normal clinical settings. The need for informed consent was waived due to the retrospective and observational design of the study and the use of secondary data. The original data collection had been approved by the Ethics Committee of the Japanese Association of Rehabilitation Medicine (November 14, 2014). However, the committee did not issue an approval number. The inclusion criteria were as follows: age, 20 - 100 years; days from onset to admission, 20 - 200 days; length of ward stay, 1 - 255 days; and gain (the difference between each m-FIM item score at discharge and admission), $>0$. All data were collected, and finally, the data of 3034 cases were analyzed. These data were classified into three groups according to m-FIM score upon admission: the severe $(<39)$, moderate $(<78)$, and slight (more than 78 ) group, according to m-FIM score at admission. m-FIM scores at admission and discharge were the primary outcome measures. Similarly, information based on the National Institutes of Health Stroke Scale (NIHSS) was collected. A flowchart of the participation of children in the study is shown in Figure 1. The FIM score is reported to have high reliability and validity concerning the ADL evaluation after stroke; it consists of $13 \mathrm{~m}$-FIM and 5 cognitive FIM (c-FIM) items 


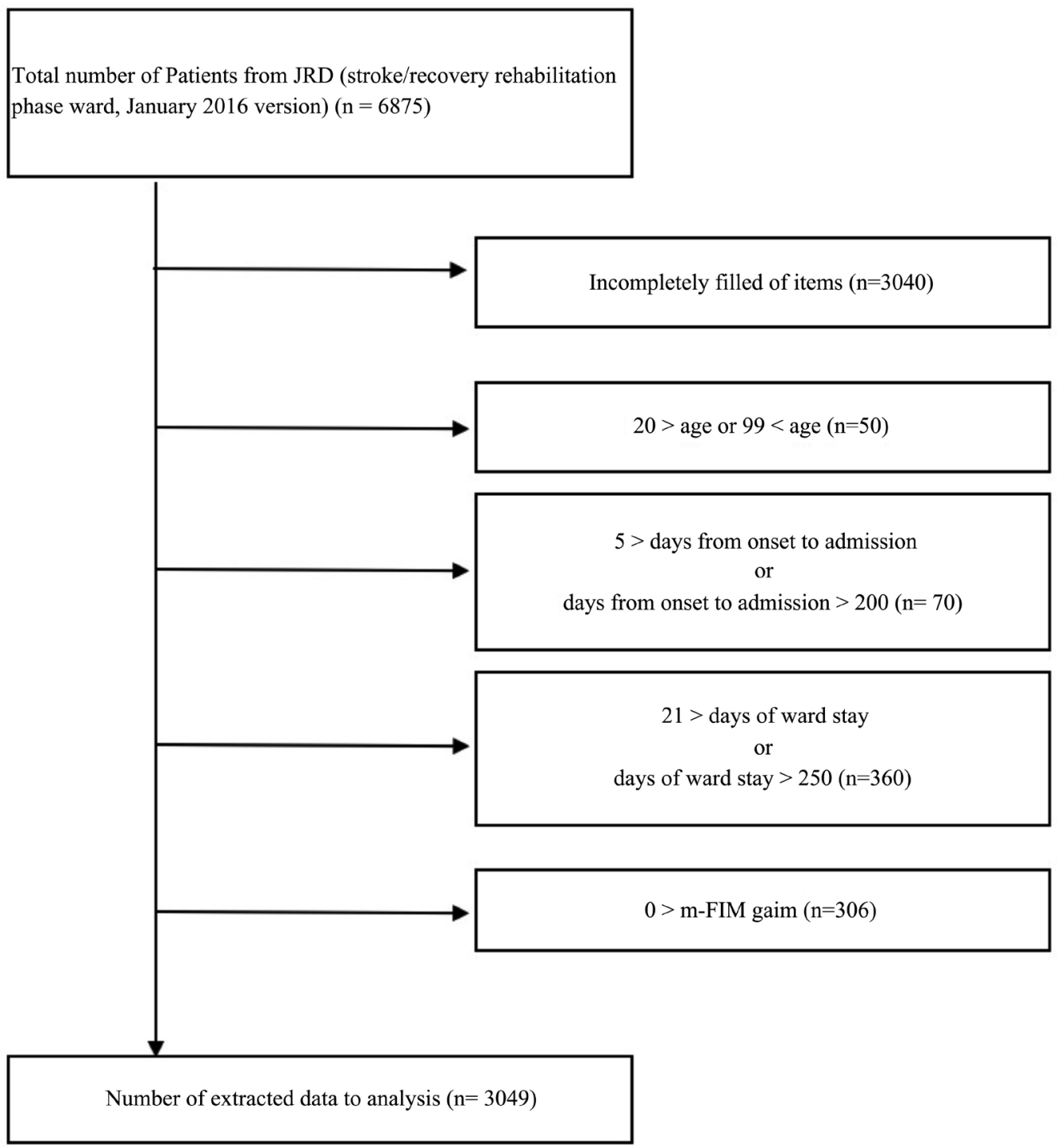

Figure 1. Flowchart of the data sampling with reasons for exclusion. JRD, Japanese Rehabilitation Database; FIM, Functional Independence Measure; gain, the difference between each motor FIM at discharge and admission.

[18] [19]. The m-FIM comprises 13 items in 4 subscales: Self-care, Sphincter control, Transfers, and Locomotion. Self-care includes the activities of eating, grooming, bathing, dressing (upper body [U/B] and lower body [L/B]), Toileting, Sphincter control (Bladder and Bowel management), Transfers (Transferring to bed, chair, wheelchair/toilet/, and tub/shower), and Locomotion (Walk or Wheelchair, and Stairs). All items were scored using a 7-point ordinal scale: level 1, Total Assist; level 2, Maximal Assist; level 3, Moderate Assist; level 4, Minimal Assist; level 5, Supervision; level 6, Modified Independence; and level 7, Complete Independence. Sex differences were not significant, and reports on such differences are limited [20] [21]. The stroke type does not generally influence the prognosis [22] [23]. Therefore, this information was excluded from this study. Age, the duration from the onset to hospital admission, the length of ward 
stay, the cognitive FIM score, and the NIHSS at admission were set as the general items.

\subsection{Statistical Analysis}

First, all data were modified as mean-centered values to avoid multicollinearity among items. The mean-centering value was calculated by subtracting the mean from the raw value. Second, correlation analyses were performed, and the evaluation of locomotion interaction was subsequently performed using hierarchical multiple regression. Hierarchical regression is a way to show if targeted variables show a statistically significant amount of variance in a dependent variable after accounting for all other variables. In this way, several regression models are built by adding variables to a previous model at each step. This method allows for the examination of the contribution above and beyond the first group of independent variables. In this study, the NIHSS and c-FIM scores were set as the control variables. This analysis used the m-FIM gain of self-care as the dependent variable in a prediction formula (model 1) using two items as independent variables (NIHSS, c-FIM) to evaluate the correlation of the control variables. Model 2 added two items as independent variables (gain of locomotion and gain of an item with the closest correlation coefficient to the dependent variable [Spearman's rank correlation]). An interaction was considered to occur when the effect of an independent variable on a dependent variable varied across the levels of a moderating variable. Locomotion gain (the difference between the locomotion item score at discharge and admission) was set as the moderating variable in this study [24] [25]. Consequently, a prediction formula, namely "model 3", added a mean-centering value to model 2. Finally, a simple slope analysis was performed as a subtest to assess locomotion interaction following the method by Cohen $\&$ Cohen [25] and the Johnson-Neyman technique (JN technique) [26]. In the concept of interaction, the idea of a two-way effect is essential, as opposed to a causal one-way effect. The simple slope method evaluates whether the influence of the independent variable on the dependent variable is statistically significant when the adjustment variable takes a specific value, and has been reported as an effective procedure for investigating the significance of an interaction. That is, fixed values of the moderator are chosen, and the significance of X's effect is investigated at those points with a hypothesis test or by constructing a confidence interval. However, when the moderator value is continuous, a better approach is the JN technique. Rather than testing for significance at fixed values of the moderator, the JN technique solves for the values of the moderator for which the effect of $\mathrm{X}$ on $\mathrm{Y}$ becomes or ceases to be significant. Moreover, it can express, as a function of the moderator, the lower and upper bounds for the confidence bands estimating the effect of X on Y. A graph of the confidence bands makes it easy to see for which values of the moderator the effect of the focal predictor on the response is significant. The statistical significance level was set at $\mathrm{p}<0.05$. Statistical analyses were conducted using IBM SPSS version 20.0 (IBM Corp., Armonk, NY, USA), and the online software created by Preacher, Curran, \& Bauer 
was used for simple slope analysis.

\section{Results}

The baseline characteristics of subjects in each group are shown in Table 1 . Multiple comparisons of four items as general information showed a significance except "days from the onset" $(\mathrm{p}<0.01)$. Table 2 shows the details of the correlation among all items. Locomotion gain correlated with other self-care; in the slight group, the correlation was shown only with toileting and bowel management. In the other two groups, a hierarchical multiple regression showed an interaction as follows: in the severe group, grooming gain, dressing [U/B] gain, and bladder management gain, and in the moderate group, dressing [U/B] gain (Table 3). Figure 2 illustrates the interactions, i.e., the relationship between the independent and dependent values under moderating value variation (means \pm 1 $\mathrm{SD})$. In the severe patient group, the simple slope was significant for three self-care items (grooming gain, dressing [L/B] gain, and bladder management gain). Only dressing [U/B] showed significance in the moderate group; however, it has been reported that a subtest of interaction was limited in the case of a continuous variable. For this reason, Figure 3 presents a region of significance and $95 \%$ confidence band of simple slope in each group. The value of locomotion gain range was relevant to under the lower or over the upper bound region in all items, and it was significant.

Table 1. Descriptive characteristics of the subjects in each group.

\begin{tabular}{|c|c|c|c|}
\hline & $\begin{array}{l}\text { Severe group } \\
(\mathrm{n}=1383)\end{array}$ & $\begin{array}{l}\text { Moderate group } \\
\qquad(\mathrm{n}=1335)\end{array}$ & $\begin{array}{l}\text { Slight group } \\
\quad(\mathrm{n}=134)\end{array}$ \\
\hline Age & $72.79 \pm 11.86$ & $69.71 \pm 12.51$ & $63.38 \pm 12.41$ \\
\hline Days form onset to admission & $34.36 \pm 24.18$ & $34.57 \pm 18.61$ & $33.71 \pm 18.73$ \\
\hline Days of ward stay & $112.03 \pm 49.30$ & $87.48 \pm 43.28$ & $51.18 \pm 33.22$ \\
\hline NIHSS at admission & $4.28 \pm 6.73$ & $1.24 \pm 2.60$ & $0.42 \pm 0.95$ \\
\hline cFIM at admission & $15.07 \pm 8.02$ & $25.75 \pm 6.75$ & $30.25 \pm 5.53$ \\
\hline t-FIM score at admission & $35.73 \pm 13.58$ & $83.57 \pm 14.74$ & $115.07 \pm 7.19$ \\
\hline t-FIM score at discharge & $65.96 \pm 31.43$ & $106.61 \pm 14.18$ & $120.66 \pm 5.44$ \\
\hline Eating gain & $1.73 \pm 1.87$ & $0.64 \pm 0.99$ & $0.12 \pm 0.47$ \\
\hline Grooming gain & $1.98 \pm 1.93$ & $1.18 \pm 1.16$ & $0.10 \pm 0.33$ \\
\hline Bathing gain & $1.54 \pm 1.79$ & $1.77 \pm 1.59$ & $0.50 \pm 0.96$ \\
\hline Dressing upper body gain & $2.17 \pm 2.08$ & $1.77 \pm 1.53$ & $0.14 \pm 0.52$ \\
\hline Dressing lower body gain & $2.05 \pm 2.13$ & $1.93 \pm 1.59$ & $0.15 \pm 0.51$ \\
\hline Toileting gain & $2.31 \pm 2.17$ & $1.57 \pm 1.31$ & $0.17 \pm 0.46$ \\
\hline Bladder management gain & $2.00 \pm 2.19$ & $0.91 \pm 1.29$ & $0.05 \pm 0.25$ \\
\hline Bowel management gain & $2.03 \pm 2.09$ & $0.74 \pm 1.11$ & $0.06 \pm 0.33$ \\
\hline Locomotion gain & $2.22 \pm 2.14$ & $2.17 \pm 1.81$ & $0.42 \pm 0.70$ \\
\hline
\end{tabular}

c-FIM, cognitive Functional Independent Measure; t-FIM, total Functional Independent Measure; gain, the difference between each motor FIM at discharge and admission. 
Table 2. Correlation among all items in each group after mean-centering. (a) Severe group; (b) Moderate group; (c) Slight group. (a)

\begin{tabular}{|c|c|c|c|c|c|c|c|c|c|c|c|c|c|c|}
\hline & $\stackrel{\infty}{4}$ & 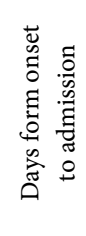 & 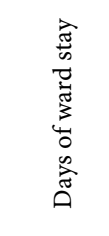 & 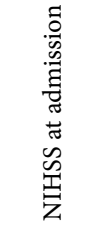 & 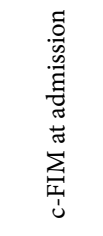 & 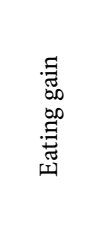 & 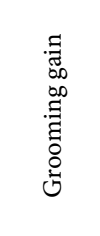 & 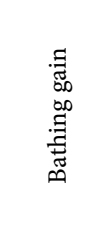 & 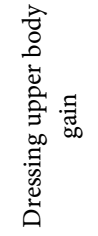 & 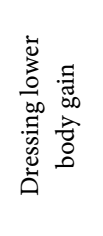 & 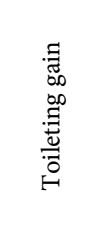 & 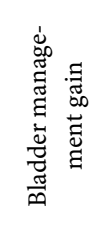 & 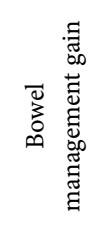 & 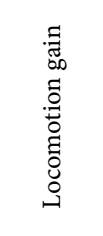 \\
\hline Age & 1.00 & -0.049 & $-0.152^{* *}$ & +0.023 & $-0.088^{* *}$ & $-0.125^{\star *}$ & ${ }^{*}-0.227^{\star *}$ & * $-0.300^{* *}$ & $-0.287^{* *}$ & $-0.288^{* *}$ & $-0.273^{* *}$ & * $-0.307^{\star *}$ & ${ }^{*}-0.253^{* *}$ & $-0.324^{* *}$ \\
\hline Days form onset to admission & & 1.00 & $0.079^{* *}$ & $-0.303^{*}$ & * $-0.113^{* *}$ & $-0.284^{* *}$ & ${ }^{*}-0.286^{* *}$ & * $-0.271^{* *}$ & $-0.260^{* *}$ & $-0.258^{* \star}$ & $-0.277^{\star *}$ & $\star-0.215^{\star *}$ & ${ }^{*}-0.227^{\star *}$ & $-0.243^{\star *}$ \\
\hline Days of ward stay & & & 1.00 & $0.064^{*}$ & 0.031 & $0.066^{*}$ & 0.048 & -0.003 & $0.071^{\star *}$ & 0.043 & $0.072^{\star *}$ & $0.086^{* *}$ & $0.092^{\star *}$ & $0.099^{* *}$ \\
\hline NIHSS at admission & & & & 1.00 & $-0.257^{\star *}$ & $0.161^{\star *}$ & $0.066^{*}$ & 0.028 & -0.008 & -0.013 & -0.021 & 0.000 & 0.002 & -0.019 \\
\hline c-FIM at admission & & & & & 1.00 & 0.017 & $0.249^{* *}$ & $0.340^{* *}$ & $0.351^{\star *}$ & $0.362^{\star \star}$ & $0.372^{\star *}$ & $0.310^{* *}$ & $0.258^{\star *}$ & $0.325^{* *}$ \\
\hline Eating gain & & & & & & 1.00 & $0.611^{* *}$ & $0.422^{* *}$ & $0.443^{* *}$ & $0.410^{* *}$ & $0.415^{* *}$ & $0.402^{* *}$ & $0.431^{\star *}$ & $0.368^{* *}$ \\
\hline Grooming gain & & & & & & & 1.00 & $0.680^{* \star}$ & $0.733^{* *}$ & $0.709^{* \star}$ & $0.694^{* *}$ & $0.601^{* *}$ & $0.626^{\star *}$ & $0.624^{\star *}$ \\
\hline Bathing gain & & & & & & & & 1.00 & $0.795^{* *}$ & $0.801^{* *}$ & $0.777^{* *}$ & $0.631^{* *}$ & $0.594^{\star *}$ & $0.674^{* *}$ \\
\hline Dressing upper body gain & & & & & & & & & 1.00 & $0.939^{* *}$ & $0.812^{* *}$ & $0.684^{* *}$ & $0.649^{* *}$ & $0.703^{* *}$ \\
\hline Dressing lower body gain & & & & & & & & & & 1.00 & $0.841^{* *}$ & $0.688^{* *}$ & $0.642^{\star *}$ & $0.694^{* *}$ \\
\hline Toileting gain & & & & & & & & & & & 1.00 & $0.741^{\star *}$ & $0.707^{\star *}$ & $0.710^{* *}$ \\
\hline Bladder management gain & & & & & & & & & & & & 1.00 & $0.831^{* *}$ & $0.607^{* *}$ \\
\hline Bowel management gain & & & & & & & & & & & & & 1.00 & $0.592^{* *}$ \\
\hline Locomotion gain & & & & & & & & & & & & & & 1.00 \\
\hline
\end{tabular}

(b)

\begin{tabular}{|c|c|c|c|c|c|c|c|c|c|c|c|c|c|c|}
\hline & $\ddot{\square}$ & 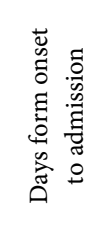 & 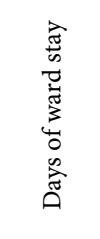 & 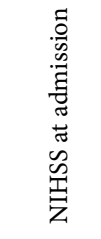 & 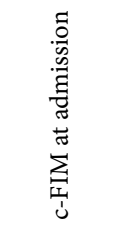 & $\begin{array}{l}\text { 泀 } \\
\text { o. } \\
\text { o. } \\
\text { 焉 }\end{array}$ & $\begin{array}{l}\cdot \tilde{\Xi} \\
\infty \\
00 \\
.0 \\
. \\
0 \\
0 \\
0 \\
0\end{array}$ & 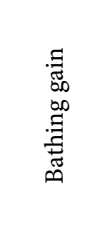 & 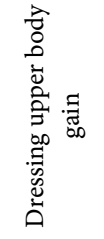 & 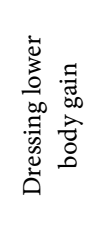 & 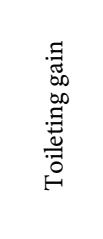 & 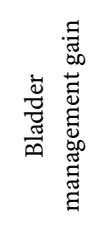 & 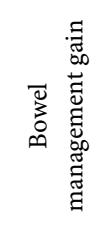 & 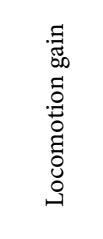 \\
\hline Age & 1.00 & $-0.135^{* *}$ & $-0.117^{* *}$ & * -0.013 & $-0.153^{* *}$ & -0.023 & 0.003 & $-0.133^{* *}$ & $+0.056^{*}$ & -0.029 & $-0.093^{* *}$ & +0.040 & 0.042 & $-0.101^{* *}$ \\
\hline Days form onset to admission & & 1.00 & $0.222^{* *}$ & $-0.096^{* *}$ & ${ }^{*}-0.100^{\star *}$ & ${ }^{*}-0.086^{\star *}$ & $-0.100^{* \star}$ & $\star-0.122^{\star *}$ & ${ }^{*}-0.086^{* *}$ & $-0.073^{* *}$ & +0.032 & -0.004 & 0.026 & -0.034 \\
\hline Days of ward stay & & & 1.00 & $0.105^{\star *}$ & $-0.108^{\star *}$ & +0.031 & 0.050 & -0.022 & $0.162^{\star *}$ & $0.213^{* *}$ & $0.317^{\star *}$ & $0.155^{\star *}$ & $0.156^{* *}$ & $0.223^{* *}$ \\
\hline NIHSS at admission & & & & 1.00 & $-0.139^{* *}$ & $+0.059^{*}$ & $0.059^{*}$ & -0.011 & $0.101^{\star *}$ & $0.120^{* *}$ & $0.106^{* *}$ & 0.043 & 0.038 & 0.042 \\
\hline c-FIM at admission & & & & & 1.00 & $-0.103^{* *}$ & $-0.125^{* *}$ & ${ }^{\star} 0.077^{\star *}$ & -0.047 & -0.027 & -0.018 & $-0.261^{\star *}$ & ${ }^{*}-0.228^{* *}$ & * 0.047 \\
\hline Eating gain & & & & & & 1.00 & $0.344^{* *}$ & $0.210^{* *}$ & $0.177^{\star *}$ & $0.161^{* *}$ & $0.202^{* *}$ & $0.138^{\star *}$ & $0.110^{* *}$ & $0.123^{* *}$ \\
\hline Grooming gain & & & & & & & 1.00 & $0.329^{* *}$ & $0.431^{\star *}$ & $0.422^{* *}$ & $0.323^{* *}$ & $0.223^{\star *}$ & $0.191^{\star *}$ & $0.207^{* *}$ \\
\hline Bathing gain & & & & & & & & 1.00 & $0.333^{\star *}$ & $0.313^{* *}$ & $0.217^{\star *}$ & $0.113^{* *}$ & $0.108^{\star *}$ & $0.150^{\star *}$ \\
\hline Dressing upper body gain & & & & & & & & & 1.00 & $0.869^{* *}$ & $0.417^{* *}$ & $0.212^{\star *}$ & $0.206^{\star *}$ & $0.276^{\star *}$ \\
\hline Dressing lower body gain & & & & & & & & & & 1.00 & $0.481^{\star \star}$ & $0.216^{\star *}$ & $0.208^{\star *}$ & $0.300^{\star *}$ \\
\hline Toileting gain & & & & & & & & & & & 1.00 & $0.254^{* *}$ & $0.274^{\star *}$ & $0.371^{\star *}$ \\
\hline Bladder management gain & & & & & & & & & & & & 1.00 & $0.678^{* *}$ & $0.101^{\star *}$ \\
\hline Bowel management gain & & & & & & & & & & & & & 1.00 & $0.087^{* *}$ \\
\hline Locomotion gain & & & & & & & & & & & & & & 1.00 \\
\hline
\end{tabular}


(c)

\begin{tabular}{|c|c|c|c|c|c|c|c|c|c|c|c|c|c|c|}
\hline & 茫 & 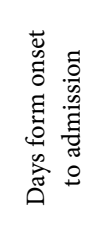 & 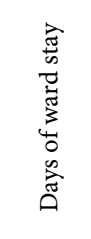 & 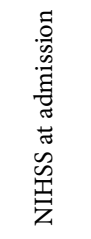 & 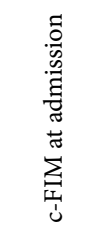 & 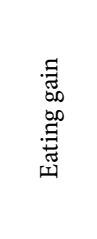 & 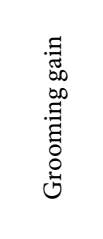 & 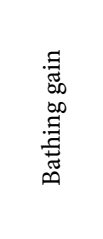 & 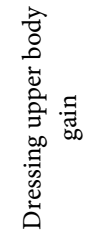 & 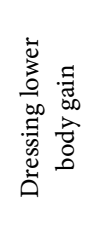 & 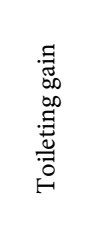 & 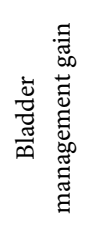 & 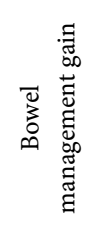 & 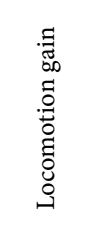 \\
\hline Age & 1.00 & $-0.133^{\star}$ & -0.058 & -0.056 & -0.069 & 0.009 & -0.018 & -0.044 & -0.054 & -0.092 & 0.093 & 0.014 & -0.058 & 0.105 \\
\hline Days form onset to admission & & 1 & $0.126^{*}$ & -0.002 & $-0.122^{*}$ & 0.005 & -0.040 & -0.027 & -0.004 & -0.001 & -0.035 & 0.034 & 0.005 & -0.107 \\
\hline Days of ward stay & & & 1 & 0.066 & $-0.284^{* *}$ & -0.002 & 0.056 & 0.039 & 0.070 & 0.057 & 0.100 & $0.141^{*}$ & $0.158^{* *}$ & 0.088 \\
\hline NIHSS at admission & & & & 1 & $-0.194^{\star *}$ & 0.042 & $0.149^{* *}$ & 0.084 & 0.098 & $0.117^{*}$ & 0.063 & 0.029 & -0.067 & 0.059 \\
\hline c-FIM at admission & & & & & 1 & $-0.118^{*}$ & $-0.205^{\star *}$ & $-0.108^{*}$ & $-0.115^{*}$ & $-0.110^{*}$ & -0.078 & -0.045 & -0.056 & 0.065 \\
\hline Eating gain & & & & & & 1 & $0.268^{\star *}$ & 0.034 & $0.116^{*}$ & $0.126^{*}$ & 0.046 & 0.027 & 0.071 & -0.025 \\
\hline Grooming gain & & & & & & & 1 & $0.161^{* *}$ & $0.322^{* *}$ & $0.306^{* *}$ & $0.269^{* *}$ & -0.020 & -0.023 & 0.059 \\
\hline Bathing gain & & & & & & & & 1 & $0.142^{* *}$ & $0.111^{*}$ & 0.107 & 0.088 & 0.005 & -0.010 \\
\hline Dressing upper body gain & & & & & & & & & 1 & $0.905^{* *}$ & $0.120^{*}$ & -0.004 & -0.010 & 0.034 \\
\hline Dressing lower body gain & & & & & & & & & & 1 & $0.154^{* *}$ & 0.016 & -0.014 & 0.058 \\
\hline Toileting gain & & & & & & & & & & & 1 & 0.035 & -0.003 & $0.205^{* *}$ \\
\hline Bladder management gain & & & & & & & & & & & & 1 & $0.510^{* *}$ & 0.005 \\
\hline Bowel management gain & & & & & & & & & & & & & 1.00 & $0.087^{\star *}$ \\
\hline Locomotion gain & & & & & & & & & & & & & & 1.00 \\
\hline
\end{tabular}

NIHSS, National Institutes of Health Stroke Scale; c-FIM, cognitive Functional Independent Measure; gain, the difference between each motor FIM at discharge and admission. ${ }^{*}: \mathrm{p}<0.05,{ }^{* *}$ : $\mathrm{p}<0.01$.

Table 3. The results of a hierarchical multiple regression analysis in each group. (a) Severe group; (b) Moderate group.

(a)

\begin{tabular}{|c|c|c|c|c|c|c|c|c|c|}
\hline Grooming gain & & & & & & & & & \\
\hline \multirow{2}{*}{ Variable } & \multicolumn{3}{|c|}{ Step 1} & \multicolumn{3}{|c|}{ Step 2} & \multicolumn{3}{|c|}{ Step 3} \\
\hline & $\mathrm{b}$ & & bSE & $\mathrm{b}$ & & bSE & $\mathrm{b}$ & & bSE \\
\hline \multicolumn{10}{|l|}{ Step 1} \\
\hline NIHSS & 0.033 & ** & 0.007 & 0.014 & $* *$ & 0.004 & 0.012 & $* *$ & 0.004 \\
\hline c-FIM & 0.083 & ** & 0.006 & 0.012 & $* *$ & 0.004 & 0.014 & ** & 0.004 \\
\hline \multicolumn{10}{|l|}{ Step 2} \\
\hline Dressing lower body gain & & & & 0.526 & $* *$ & 0.018 & 0.505 & $* *$ & 0.019 \\
\hline Locomotion gain & & & & 0.185 & $* *$ & 0.018 & 0.177 & $* *$ & 0.018 \\
\hline \multicolumn{10}{|l|}{ Step 3} \\
\hline \multicolumn{10}{|l|}{ Dressing lower body gain } \\
\hline \multicolumn{10}{|l|}{$\times$ Locomotion gain } \\
\hline$\Delta \mathrm{R}^{2}$ & & & & 0.543 & $* *$ & & 0.005 & ** & \\
\hline Adj $R^{2}$ & & & & 0.672 & & & 0.677 & & \\
\hline
\end{tabular}




\section{Continued}

\begin{tabular}{|c|c|c|c|c|c|c|c|c|c|}
\hline \multicolumn{10}{|l|}{ Dressing lower body gain } \\
\hline \multirow{2}{*}{ Variable } & \multicolumn{3}{|c|}{ Step 1} & \multicolumn{3}{|c|}{ Step 2} & \multicolumn{3}{|c|}{ Step 3} \\
\hline & $\mathrm{b}$ & & bSE & $\mathrm{b}$ & & bSE & $\mathrm{b}$ & & bSE \\
\hline \multicolumn{10}{|l|}{ Step 1} \\
\hline NIHSS & 0.027 & $* *$ & 0.008 & 0.001 & & 0.003 & 0.001 & & 0.003 \\
\hline cFIM & 0.102 & $* *$ & 0.007 & 0.009 & $* *$ & 0.003 & 0.009 & $* *$ & 0.003 \\
\hline \multicolumn{10}{|l|}{ Step 2} \\
\hline Dressing upper body gain & & & & 0.908 & $* *$ & 0.013 & 0.902 & $* *$ & 0.014 \\
\hline Locomotion gain & & & & 0.062 & ** & 0.013 & 0.058 & $* *$ & 0.013 \\
\hline \multicolumn{10}{|l|}{ Step 3} \\
\hline \multicolumn{6}{|l|}{ Dressing upper body gain } & & 0.015 & $* *$ & 0.005 \\
\hline$\Delta \mathrm{R}^{2}$ & & & & 0.747 & $* *$ & & 0.001 & $* *$ & \\
\hline Adj $R^{2}$ & & & & 0.885 & & & 0.886 & & \\
\hline \multicolumn{10}{|l|}{ Bladder management gain } \\
\hline \multirow{2}{*}{ Variable } & \multicolumn{3}{|c|}{ Step 1} & \multicolumn{3}{|c|}{ Step 2} & \multicolumn{3}{|c|}{ Step 3} \\
\hline & $\mathrm{b}$ & & bSE & $\mathrm{b}$ & & bSE & $\mathrm{b}$ & & bSE \\
\hline \multicolumn{10}{|l|}{ Step 1} \\
\hline NIHSS & 0.028 & $* *$ & 0.009 & 0.007 & & 0.005 & 0.006 & & 0.005 \\
\hline c-FIM & 0.090 & $* *$ & 0.007 & 0.022 & $* *$ & 0.004 & 0.024 & $* *$ & 0.004 \\
\hline \multicolumn{10}{|l|}{ Step 2} \\
\hline Bowel management gain & & & & 0.750 & $* *$ & 0.019 & 0.739 & $* *$ & 0.019 \\
\hline Locomotion gain & & & & 0.159 & $* *$ & 0.019 & 0.155 & $* *$ & 0.019 \\
\hline \multicolumn{10}{|l|}{ Step 3} \\
\hline $\begin{array}{l}\text { Bowel management gain } \\
\times \text { Locomotion gain }\end{array}$ & & & & & & & 0.019 & $* *$ & 0.008 \\
\hline$\Delta \mathrm{R}^{2}$ & & & & 0.613 & $* *$ & & 0.001 & $* *$ & \\
\hline Adj $R^{2}$ & & & & 0.715 & & & 0.716 & & \\
\hline
\end{tabular}

(b)

\begin{tabular}{|c|c|c|c|c|c|c|c|c|c|}
\hline Dressing upper body gain & & & & & & & & & \\
\hline \multirow{2}{*}{ Variable } & \multicolumn{3}{|c|}{ Step 1} & \multicolumn{3}{|c|}{ Step 2} & \multicolumn{3}{|c|}{ Step 3} \\
\hline & $\mathrm{b}$ & & bSE & $\mathrm{b}$ & & bSE & $\mathrm{b}$ & & bSE \\
\hline \multicolumn{10}{|l|}{ Step 1} \\
\hline NIHSS & 0.057 & $* *$ & 0.016 & -0.004 & & 0.008 & -0.004 & & 0.008 \\
\hline c-FIM & -0.008 & & 0.006 & -0.006 & & 0.003 & -0.005 & & 0.003 \\
\hline \multicolumn{10}{|l|}{ Step 2} \\
\hline Dressing lower body gain & & & & 0.830 & $* *$ & 0.014 & 0.827 & $* *$ & 0.014 \\
\hline Locomotion gain & & & & 0.015 & & 0.012 & 0.011 & & 0.012 \\
\hline \multicolumn{10}{|l|}{ Step 3} \\
\hline Dressing lower body gain & & & & & & & 0.025 & $* *$ & 0.007 \\
\hline
\end{tabular}




\section{Continued}

$\times$ Locomotion gain
$\Delta \mathrm{R}^{2}$
0.744
$0.002 \quad * *$
Adj $R^{2}$
0.755
0.757

NIHSS, National Institutes of Health Stroke Scale; c-FIM, cognitive Functional Independent Measure; gain, the difference between each motor FIM at discharge and admission. ${ }^{* *}: \mathrm{p}<0.01$.

a) Severe group

a-1)

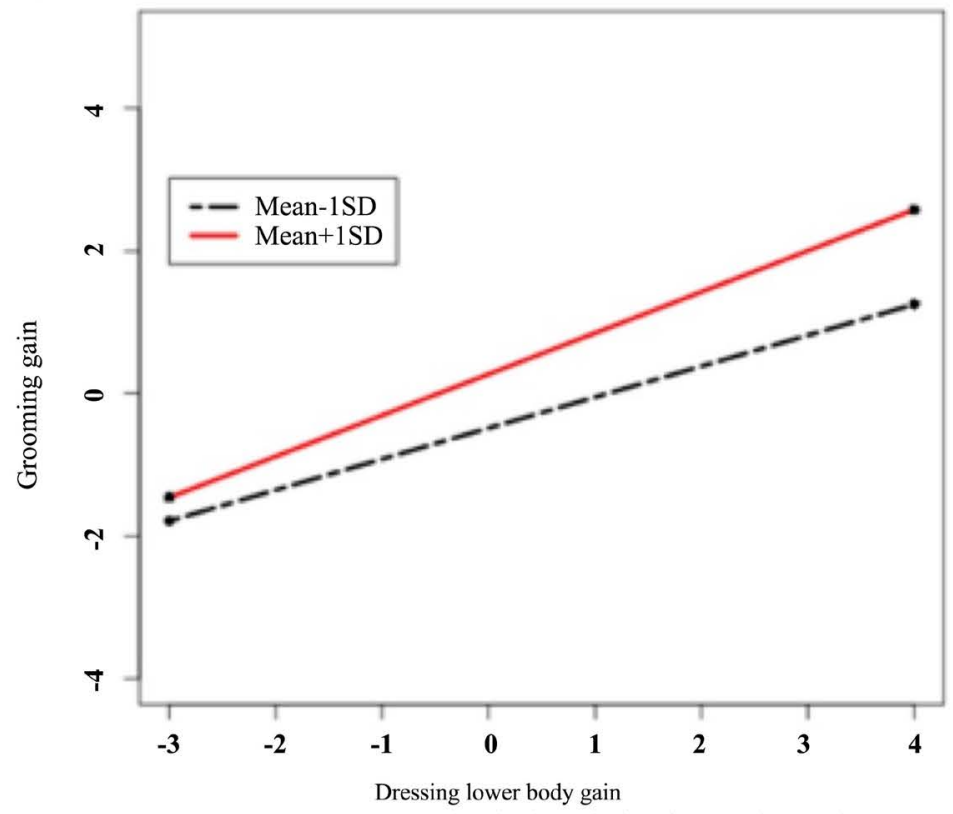

Mean - 1SD: $y=0.43 \times$ dressing lower body gain $-0.48(p<.001)$

Mean + 1SD: $y=0.48 \times$ dressing lower body gain $-0.48(\mathrm{p}<.001)$

a-2)

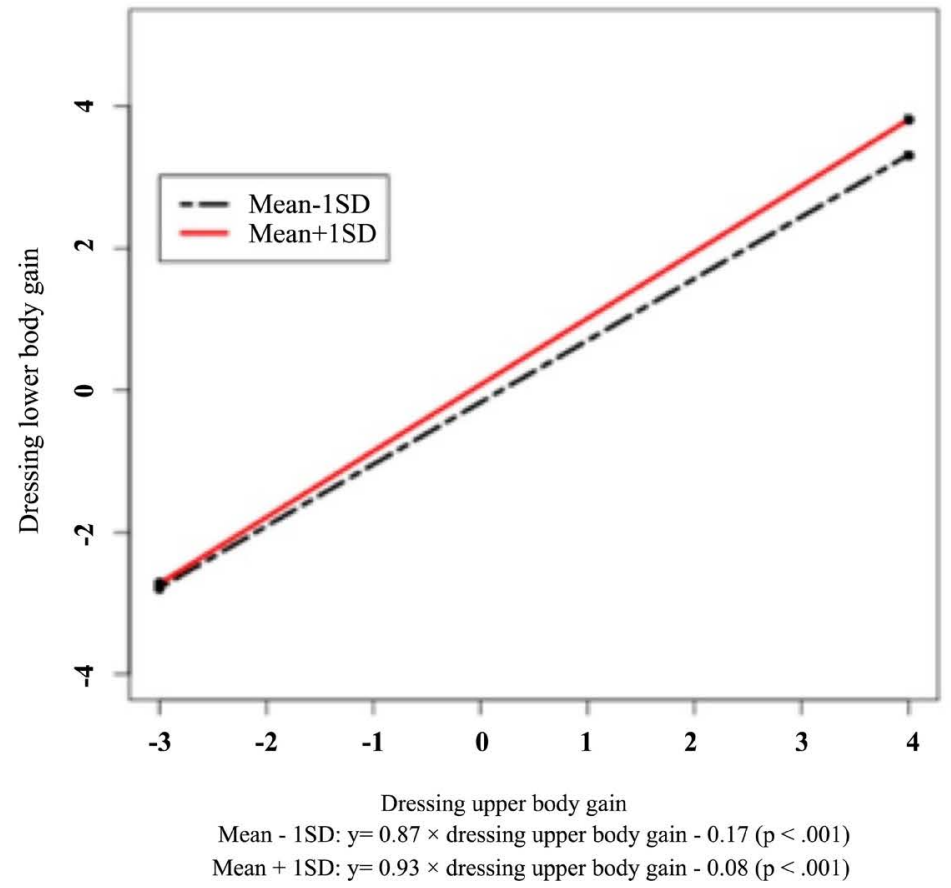




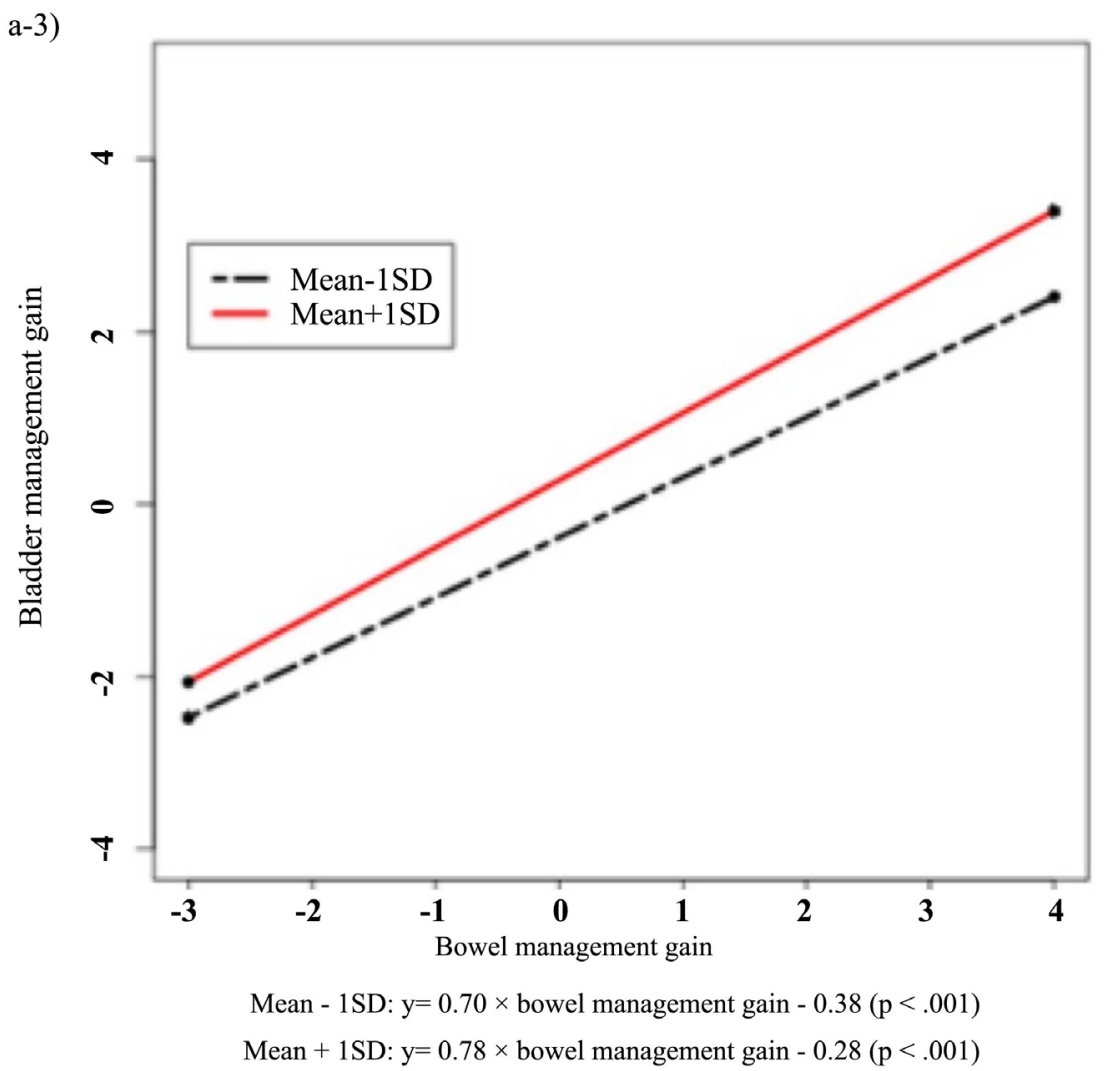

b) Moderate group

b-1)

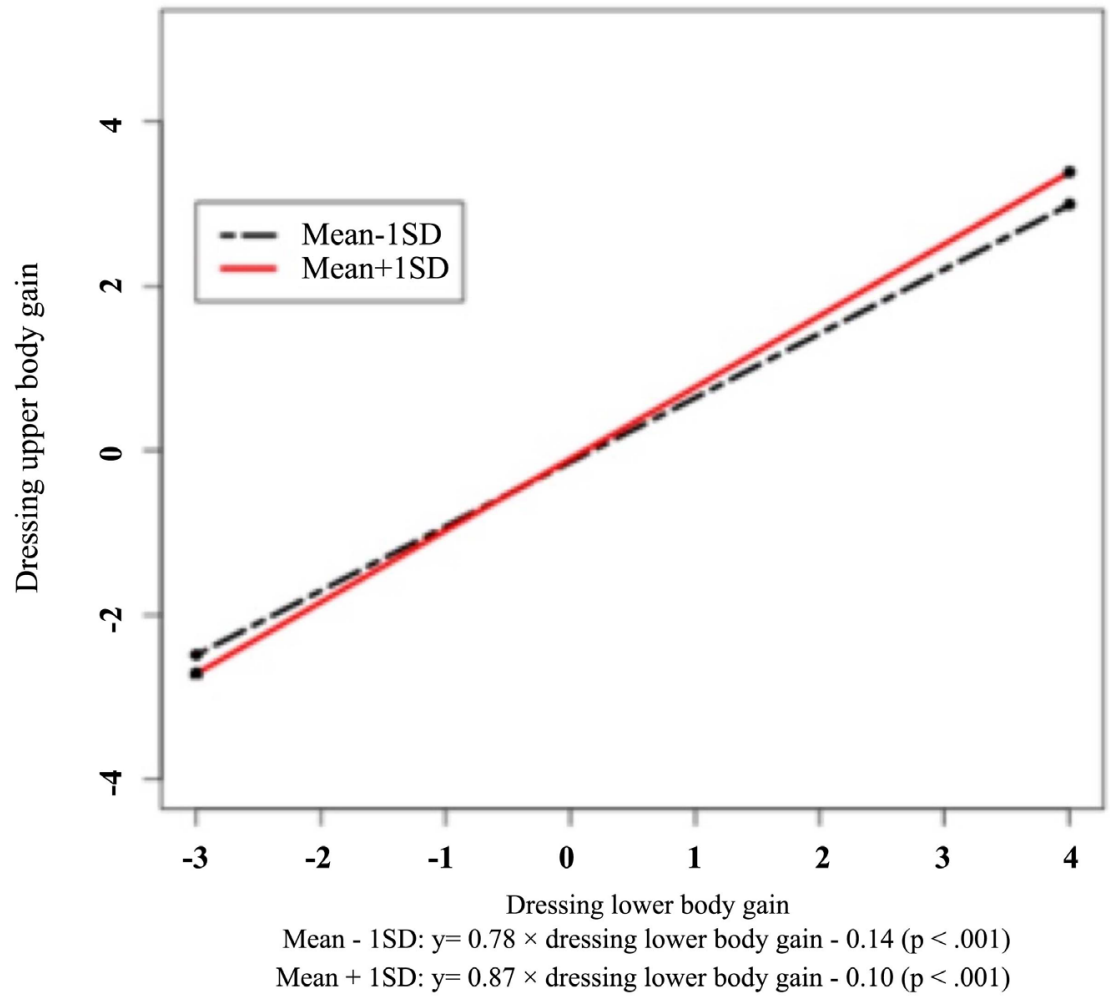

Figure 2. A result of simple slope analysis in each item and group after mean-centering gain: the difference between motor FIM at discharge and admission. 
a) Severe group

a-1) Grooming

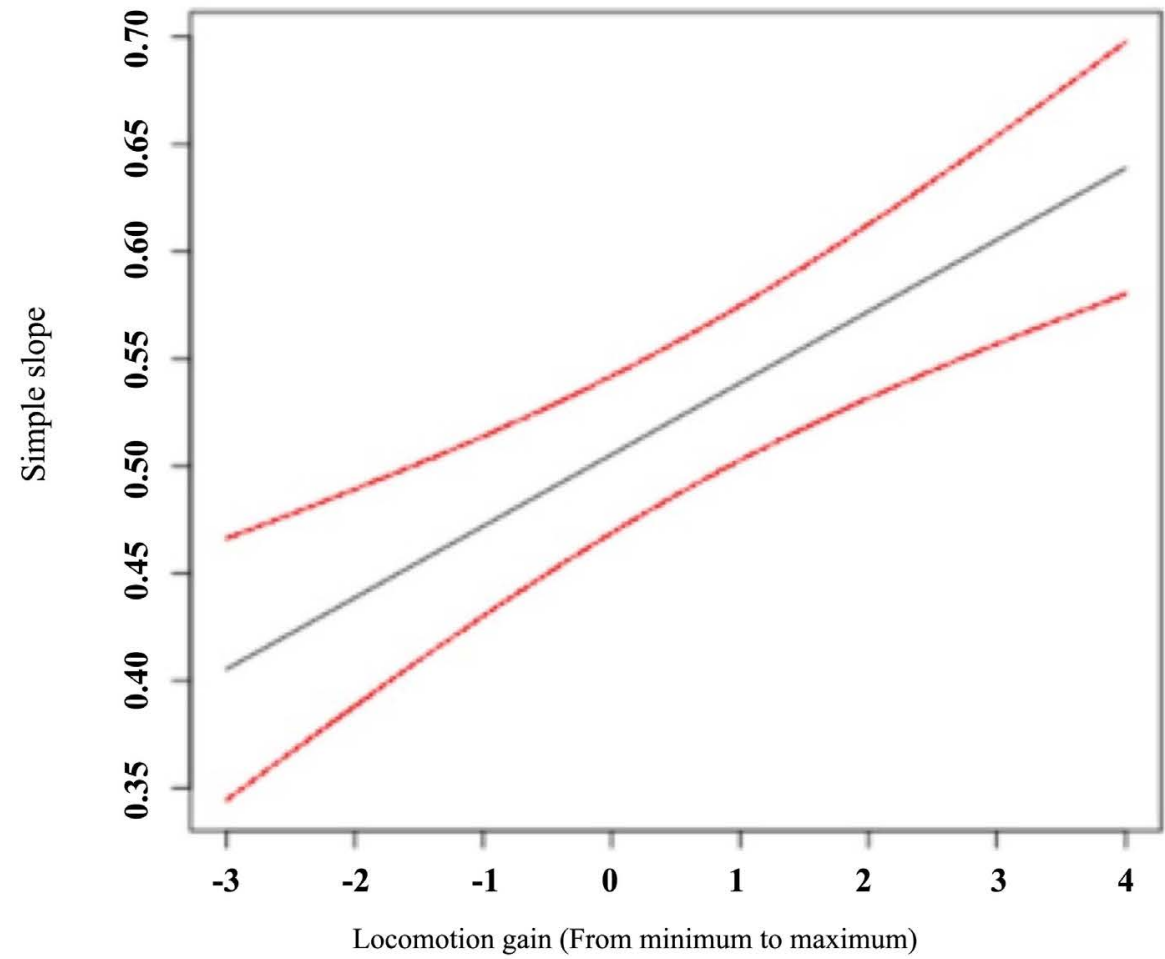

A $95 \%$ confidential interval of coefficient of dressing lower body gain to grooming gain by locomotion gain

a-2) Dressing lower body

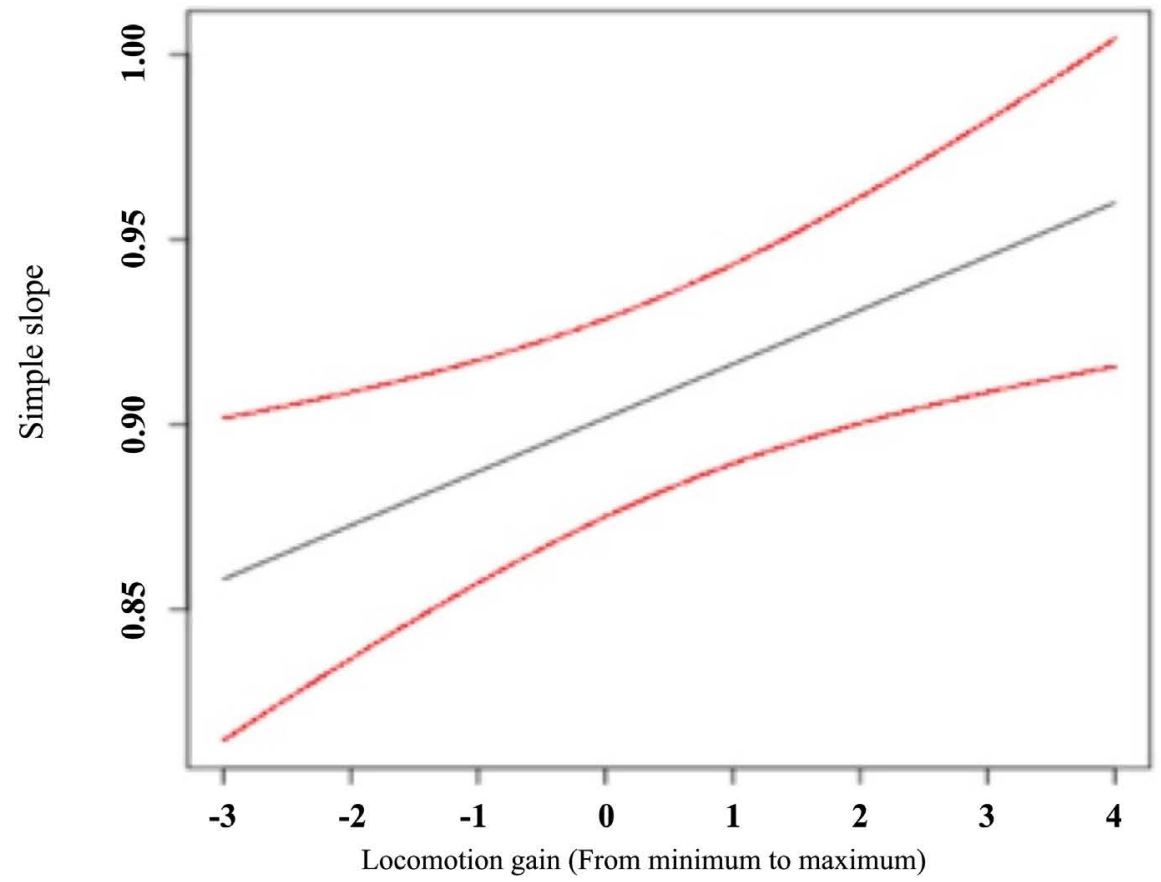

A $95 \%$ confidential interval of coefficient of dre sing upper body gain to dressing lower body by locomotion gain. 
a-3) Bladder management

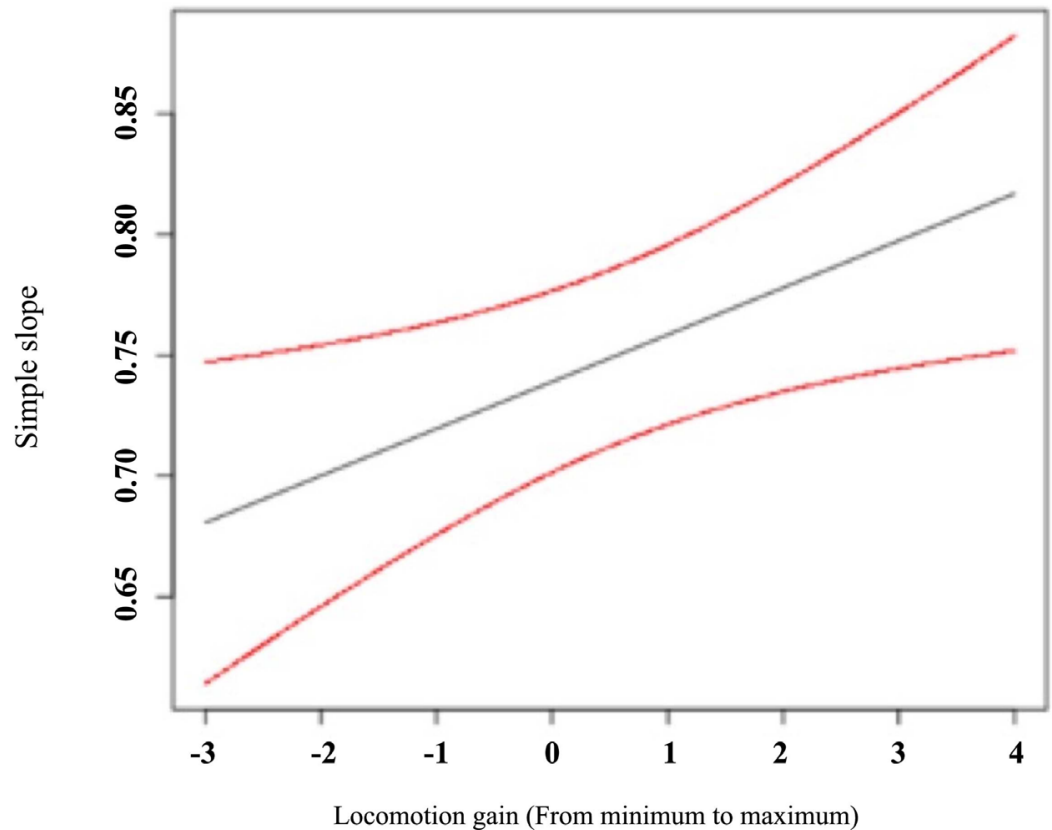

A $95 \%$ confidential interval of coefficient of bowel management gain to bladder manegment gain by locomotion gain.

b) Moderate group

b-1) Dressing upper body

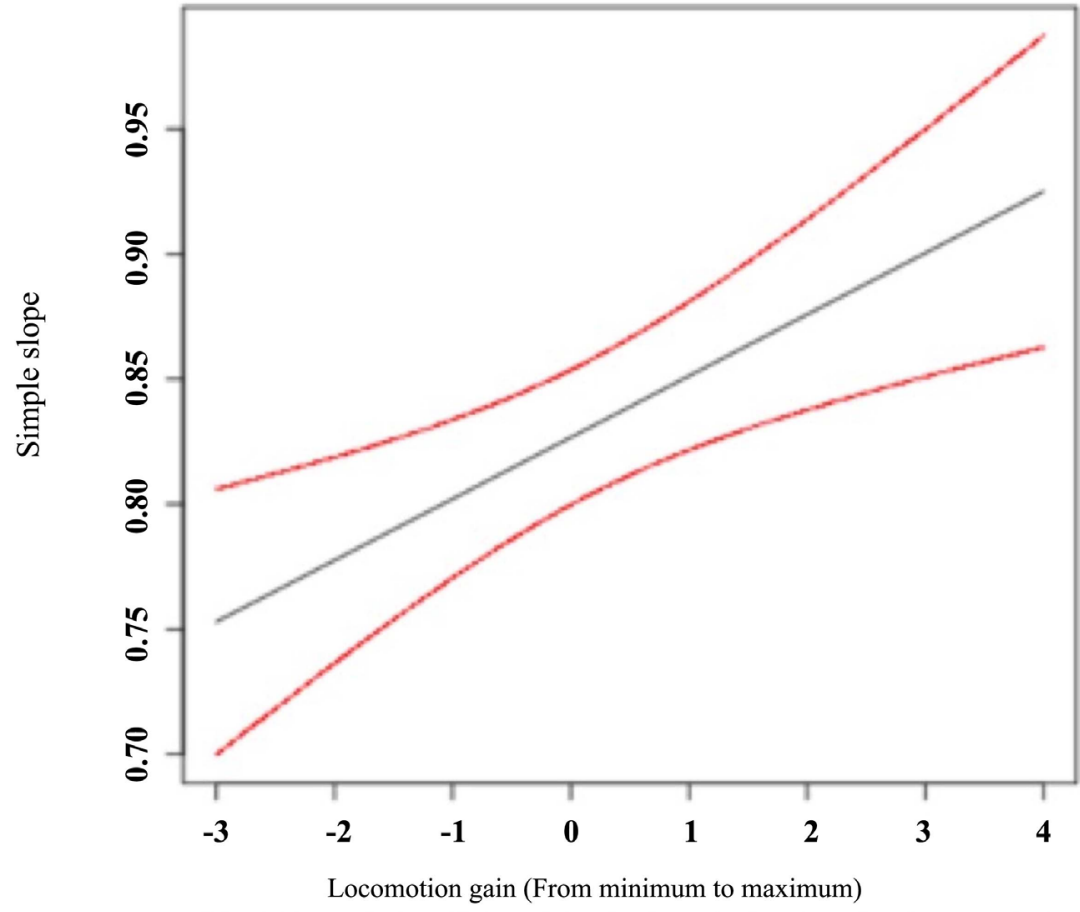

A $95 \%$ confidential interval of coefficient of dressing lower body gain to dressing upper body gair by locomotion gain.

Figure 3. A region of significance and $95 \%$ confidence band of a simple slope after mean-centering. Center line with grey color: Prediction coefficient of simple slope, up and down curve line with red color: $95 \%$ confidence interval of prediction coefficient. 


\section{Discussion}

This study investigated the interaction between locomotion and self-care in patients with stroke based on the m-FIM score at admission using multi-facility data (JRD). The main finding suggests that a variation of locomotion gain influenced improving self-care. Moreover, aspects of self-care that were influenced by locomotion interaction were as follows: for the severe group, grooming, bladder management, and dressing [L/B]; and for the moderate group, dressing [U/B]. However, locomotion interaction was not significant with any aspect of self-care in the slight group.

Previous reports have shown a correlation between ADL and gait in patients with stroke [4]. Moreover, gait training has been suggested to be important from the early phase after stroke [5] [6] [7]. It has been reported that the function of the affected side, lower extremity muscles, and balance are the major determinants of ambulation after stroke [2] [10]. Moreover, recent gait-training strategies have highlighted a relationship between the passenger and locomotion units and mentioned the importance of trunk function [2] [27]. It has been reported that the gait recovery process changed sharply from 30 to 60 points based on the total FIM (t-FIM) score, and in this study, the t-FIM score in the severe group was in this range upon admission and discharge; however, the t-FIM score at admission had already reached over 80 points after the moderate group. Moreover, self-care (except in the slight group) had a correlation with locomotion. Locomotion recovery progressed sharply from 10 to 60 points in the t-FIM score, and this range matched the t-FIM score in the severe group. Furthermore, it has been reported that with over 40 points of t-FIM in locomotion, there is a $50 \%$ probability of reaching level 5. For this reason, the influence of locomotion was assumed to be stronger in the severe group than in the other groups.

Trunk function influenced ADL improvement, and it is reported that the $\mathrm{m}$-FIM at discharge was significantly low in patients with decreased trunk function and low ADL score on admission [28] [29]. Therefore, trunk function is one of the factors that inhibit ADL. Gait training influences trunk function, and it is assumed that ADL in stroke patients with low trunk function in the severe group was influenced.

Locomotion improvement at admission was not significant compared to that at discharge in the slight group, and locomotion score was similar between admission and discharge. Furthermore, the correlation between self-care and locomotion was low in the slight group. Therefore, it was considered that locomotion interaction was not significant for self-care in the slight group.

Regarding the difficulty of various aspects of self-care, it has been reported that eating, grooming, and bowel management improved easily, unlike those of dressing [L/B] and bathing, which were most difficult [30] [31]. The aspects of self-care that showed locomotion correlation were positively related to locomotion in this study, as in previous studies; a sharply changing recovery process of extracted self-care of patients in the severe group has been reported [30] [32]. 
Eating, which is easier than grooming, was not affected by locomotion interaction. The reason for this is assumed to be that eating recovery almost reached level 5 (supervision and set-up) before the peak of locomotion recovery and had a lower correlation with locomotion than other self-care.

Both, eating and grooming, can be performed in a seated position using a backrest. However, in grooming more than in eating, it is necessary to control the center of gravity in all directions [33] [34]. Therefore, grooming is more influenced by trunk function that involves control of balance while sitting. This is considered to be the reason for the influence being observed only on grooming, and not eating. Dressing both upper and lower body showed locomotion interaction; they were strongly correlated with each other, and a similar finding was observed in this study. However, locomotion interaction was shown in different groups (with dressing [L/B] in the severe group and dressing [U/B] in the moderate group). It is necessary to control the center of gravity in all directions for dressing activity [34] [35]. Moreover, unaffected-lower limbs and trunk function have been reported to affect dressing activity independence [35]. For the reasons, it is assumed that improved gait affects more dressing $[\mathrm{L} / \mathrm{B}]$ than dressing $[\mathrm{U} / \mathrm{B}]$. Furthermore, the recovery of dressing $[\mathrm{L} / \mathrm{B}]$ was reported to change sharply from 50 to 80 in terms of the t-FIM score. In dressing [U/B], recovery progress appeared more slowly and over a wider range than dressing $[\mathrm{L} / \mathrm{B}]$ in the t-FIM score. Therefore, it was assumed that locomotion significantly influenced dressing $[\mathrm{L} / \mathrm{B}]$, even though dressing $[\mathrm{U} / \mathrm{B}]$ is commonly the first step in recovery processes. Hence, dressing $[\mathrm{L} / \mathrm{B}]$ was presented earlier than dressing $[\mathrm{U} / \mathrm{B}]$ in locomotion interaction.

Bladder and bowel management have a relationship similar to that of dressing $[\mathrm{U} / \mathrm{B}]$ and $[\mathrm{L} / \mathrm{B}]$; however, locomotion interaction was shown only for bladder management in this study. It has been reported that bowel management could reach over level 6 (supervision and set up) at less than 60 points in the t-FIM, and a peak of bowel management recovery changing was shown at a t-FIM score less than 40, before a peak of bladder management recovery changing. Moreover, bladder management recovery showed a sharp change in the range of 40 to 60 points in the t-FIM. There was a lot of overlap between a peak of bladder management recovery changing and one in the severe group in this study.

In addition, pelvic floor muscles (PFMs) are needed to control the sphincter. PFMs are related to the sphincter and support the abdominal viscera from the pelvic floor [36] [37]. PFM contractions manifest during urination, and increased abdominal pressure occurs with trunk muscle group co-contraction, e.g., the transversus abdominis, multifidus muscle, and diaphragm. These functions are affected by improved gait. Furthermore, pressure for bladder activity is lower than that for bowel activity. Therefore, bladder management was assumed to be influenced by locomotion interaction in the severe group.

In the slight group, locomotion interaction was absent in self-care. This was assumed to be caused by three reasons: correlation between locomotion and self-care was not significant, the locomotion FIM score was not significantly dif- 
ferent between admission and discharge, and FIM score average of self-care and locomotion in this group could exceed level 6.

A limitation of this study is that the NIHSS and c-FIM scores were set as the control variables in all groups without considering illness severity; therefore, the characteristics of each group were not considered in setting the control variables for that particular group. Hence, future research is needed to investigate locomotion interaction further using control variables reflecting the group characteristics.

In conclusion, this study investigated locomotion interaction and self-care in three groups that were classified based on the m-FIM upon admission. Locomotion interaction was shown mainly in the severe group based on the m-FIM at admission according to the JRD. The self-care activities that showed locomotion interaction included sitting activity. Therefore, this study's findings suggest that locomotion intervention affects sitting activities in severe cases; however, for moderate and slight cases, an intervention that not only involves locomotion training but also focuses on improvement in ADL should be considered. In the future, research should be conducted in a more representative large sample population to investigate whether gait training and ADL training correlate or not, and to further test and discuss the influencing factor of training time.

\section{Acknowledgements}

This study used the Japan Rehabilitation Database managed by the Japan Association of Rehabilitation Database, to whom we express our gratitude. The contents and conclusions of this study are not the opinions of the Association, but exclusively those of the authors.

\section{Funding}

This research received no specific grant from any funding agency in the public, commercial, or not-for-profit sectors.

\section{Conflicts of Interest}

The author declares that they have no potential conflict of interest relevant to this article to declare.

\section{References}

[1] Matsuyama, A. (2018) Factors Associated with the Walking Ability of Hemiplegic Stroke Patients. Open Journal of Nursing, 8, 14-25. https://doi.org/10.4236/ojn.2018.81002

[2] Eng, J.J. and Tang, P.F. (2007) Gait Training Strategies to Optimize Walking Ability in People with Stroke: A Synthesis of the Evidence. Expert Review of Neurotherapeutics, 7, 1417-1436. https://doi.org/10.1586/14737175.7.10.1417

[3] Adnil, W.T., Susan, H., Quinette, A.L. and Gakeemah, I.-J. (2018) An Analysis of Trunk Kinematics and Gait Parameters in People with Stroke. African Journal of Disability, 7, a310. https://doi.org/10.4102/ajod.v7i0.310 
[4] Wang, C.-H., et al. (2002) Trunk Control as an Early Predictor of Comprehensive Activities of Daily Living Function in Stroke Patients. Stroke, 33, 2626-2630. https://doi.org/10.1161/01.STR.0000033930.05931.93

[5] Cho, K.H., Lee, J.Y., Lee, K.J. and Kang, E.K. (2014) Factors Related to Gait Function in Post-Stroke Patients. Journal of Physical Therapy Science, 26, 1941-1944. https://doi.org/10.1589/jpts.26.1941

[6] Granger, C.V., Cotter, A.C., Hamilton, B.B. and Fiedler, R.C. (1993) Functional Assessment Scales: A Study of Persons after Stroke. Archives of Physical Medicine and Rehabilitation, 74, 133-138.

[7] Corrigan, J.D., Smith-Knapp, K. and Granger, C.V. (1997) Validity of the Functional Independence Measure for Persons with Traumatic Brain Injury. Archives of Physical Medicine and Rehabilitation, 78, 828-834. https://doi.org/10.1016/S0003-9993(97)90195-7

[8] Inouye, M., Kishi, K., Ikeda, Y., Takada, M., Katoh, J., et al. (2000) Prediction of Functional Outcome after Stroke Rehabilitation. American Journal of Physical Medicine and Rehabilitation, 79, 513-518. https://doi.org/10.1097/00002060-200011000-00007

[9] Hsieh, C.L., Sheu, C.F., Hsueh, I.P. and Wang, C.H. (2002) Trunk Control as an Early Predictor of Comprehensive Activities of Daily Living Function in Stroke Patients. Stroke, 33, 2626-2630. https://doi.org/10.1161/01.STR.0000033930.05931.93

[10] Haruyama, K., Kawakami, M. and Otsuka, T. (2017) Effect of Core Stability Training on Trunk Function, Standing Balance, and Mobility in Stroke Patients. Neurorehabilitation and Neural Repair, 31, 240-249. https://doi.org/10.1177/1545968316675431

[11] Kwakkel, G., van Peppen, R., Wagenaar, R.C., Wood Dauphinee, S., Richards, C., et al. (2004) Effects of Augmented Exercise Therapy Time after Stroke: A Meta-Analysis. Stroke, 35, 2529-2539. https://doi.org/10.1161/01.STR.0000143153.76460.7d

[12] Sonoda, S., Saitoh, E., Nagai, S., Kawakita, M. and Kanada, Y. (2004) Full-Time Integrated Treatment Program, a New System for Stroke Rehabilitation in Japan: Comparison with Conventional Rehabilitation. American Journal of Physical Medicine and Rehabilitation, 83, 88-93. https://doi.org/10.1097/01.PHM.0000107481.69424.E1

[13] Chen, C.C., Heinemann, A.W., Granger, C.V. and Linn, R.T. (2002) Functional Gains and Therapy Intensity during Subacute Rehabilitation: A Study of 20 Facilities. Archives of Physical Medicine and Rehabilitation, 83, 1514-1523. https://doi.org/10.1053/apmr.2002.35107

[14] Kim, B.J. and Young, C. (2015) The Relationship between Activities of Daily Living (ADL), Chronic Diseases, and Depression among Older Korean Immigrants. Educational Gerontology, 41, 417-427. https://doi.org/10.1080/03601277.2014.982006

[15] Bayot, M., Dujardin, K., Tard, C., Defebvre, L., Bonnet, C.T., et al. (2018) The Interaction between Cognition and Motor Control: A Theoretical Framework for Dual-Task Interference Effects on Posture, Gait Initiation, Gait and Turning. Neurophysiologie Clinique, 48, 361-375. https://doi.org/10.1016/j.neucli.2018.10.003

[16] Yogev-Seligmann, G., Giladi, N., Gruendlinger, L. and Hausdorff, J.M. (2013) The Contribution of Postural Control and Bilateral Coordination to the Impact of Dual Tasking on Gait. Experimental Brain Research, 226, 81-93. https://doi.org/10.1007/s00221-013-3412-9

[17] Bovonsunthonchai, S., Aung, N., Hiengkaew, V. and Tretriluxana, J. (2020) A Ran- 
domized Controlled Trial of Motor Imagery Combined with Structured Progressive Circuit Class Therapy on Gait in Stroke Survivors. Scientific Reports, 24, Article No. 6945. https://doi.org/10.1038/s41598-020-63914-8

[18] Ottenbacher, K.J., Hsu, Y., Granger, C.V. and Fiedler, R.C. (1996) The Reliability of the Functional Independence Measure: A Quantitative Review. Archives of Physical Medicine and Rehabilitation, 77, 1226-1232.

https://doi.org/10.1016/S0003-9993(96)90184-7

[19] Chen, H.F., Wu, C.Y., Lin, K.C., Chen, C.L., Huang, P.C., et al. (2013) Rasch Validation of a Combined Measure of Basic and Extended Daily Life Functioning after Stroke. Neurorehabilitation and Neural Repair, 27, 125-132. https://doi.org/10.1177/1545968312457828

[20] Appelros, P., Stegmayr, B. and Terént, A. (2010) A Review on Sex Differences in Stroke Treatment and Outcome. Acta Neurologica Scandinavica, 121, 359-369. https://doi.org/10.1111/j.1600-0404.2009.01258.x

[21] Sheth, S.A., Lee, S., Warach, S.J., Gralla, J., Jahan, R., et al. (2019) Sex Differences in Outcome after Endovascular Stroke Therapy for Acute Ischemic Stroke. Stroke, 50, 2420-2427. https://doi.org/10.1161/STROKEAHA.118.023867

[22] Lopes, P.G., Lopes, J.A., Brito, C.M., Alfieri, F.M. and Rizzo Battistella, L. (2015) Relationships of Balance, Gait Performance, and Functional Outcome in Chronic Stroke Patients: A Comparison of Left and Right Lesions. BioMed Research International, 2015, Article ID: 716042. https://doi.org/10.1155/2015/716042

[23] Jørgensen, H.S., Nakayama, H., Raaschou, H.O. and Olsen, T.S. (1995) Intracerebral Hemorrhage versus Infarction: Stroke Severity, Risk Factors, and Prognosis. Annals of Neurology, 38, 45-50. https://doi.org/10.1002/ana.410380110

[24] Brankovic, M., Kardys, I., Steyerberg, E.W., Lemeshow, S., Markovic, M., et al. (2019) Understanding of Interaction (Subgroup) Analysis in Clinical Trials. European Journal of Clinical Investigation, 49, e13145. https://doi.org/10.1111/eci.13145

[25] Andersson, U., Cuervo-Cazurra, A. and Nielsen, B.B. (2014) Explaining Interaction Effects within and across Levels of Analysis. Journal of International Business Studies, 45, 1063-1071. https://doi.org/10.1057/jibs.2014.50

[26] Liu, Y., West, S.G., Levy, R. and Aiken, L.S. (2017) Tests of Simple Slopes in Multiple Regression Models with an Interaction: Comparison of Four Approaches. Multivariate Behavioral Research, 52, 445-464. https://doi.org/10.1080/00273171.2017.1309261

[27] Di Monaco, M., Trucco, M., Di Monaco, R., Tappero, R. and Cavanna, A. (2010) The Relationship between Initial Trunk Control or Postural Balance and Inpatient Rehabilitation Outcome after Stroke: A Prospective Comparative Study. Clinical Rehabilitation, 24, 543-554. https://doi.org/10.1177/0269215509353265

[28] Kwakkel, G., Wagenaar, R.C., Kollen, B.J. and Lankhorst, G.J. (1996) Predicting Disability in Stroke-A Critical Review of the Literature. Age Ageing, 25, 479-489. https://doi.org/10.1093/ageing/25.6.479

[29] Sayaka, O., Shigeru, S., Makoto, W., Hideto, O., Kei, Y., et al. (2018) Relationship between Functional Independence Measure (FIM) Score on Admission and Influence of Inhibitive Factors in a Comprehensive Inpatient Stroke Rehabilitation Ward. Japanese Journal of Comprehensive Rehabilitation Science, 9, 59-65.

[30] Koyama, T., Matsumoto, K., Okuno, T. and Domen, K. (2006) Relationships between Independence Level of Single Motor-FIM Items and FIM-Motor Scores in Patients with Hemiplegia after Stroke: An Ordinal Logistic Moldering Study. Journal of Rehabilitation Medicine, 38, 280-286. 
https://doi.org/10.1080/16501970600731420

[31] Iwai, N., Aoyagi, Y., Tokuhisa, K., Yamamoto, J. and Shimada, T. (2011) The Gaps between Capability ADL and Performance ADL of Stroke Patients in a Convalescent Rehabilitation Ward-Based on the Functional Independence Measure. The Journal of Physical Therapy Science, 23, 333-338.

https://doi.org/10.1589/jpts.23.333

[32] Heinemann, A.W., Linacre, J.M., Wright, B.D., Hamilton, B.B. and Granger, C. (1993) Relationships between Impairment and Physical Disability as Measured by the Functional Independence Measure. Archives of Physical Medicine and Rehabilitation, 74, 566-573. https://doi.org/10.1016/0003-9993(93)90153-2

[33] Takaaki, F., Atsushi, S., Yui, T., Ryuichi, K., Takuro, O., et al. (2015) Contribution of Abdominal Muscle Strength to Various Activities of Daily Living of Stroke Patients with Mild Paralysis, The Journal of Physical Therapy Science, 27, 815-818. https://doi.org/10.1589/jpts.27.815

[34] Morgan, P. (1994) The Relationship between Sitting Balance and Mobility Outcome in Stroke. Australian Journal of Physiotherapy, 40, 91-96. https://doi.org/10.1016/S0004-9514(14)60455-4

[35] Walker, M.F. and Lincoln, N.B. (1991) Factors Influencing Dressing Performance after Stroke. Journal of Neurology, Neurosurgery and Psychiatry, 54, 699-701. https://doi.org/10.1136/jnnp.54.8.699

[36] Booth, J., Paul, L., Rafferty, D. and Macinnes, C. (2013) The Relationship between Urinary Bladder Control and Gait in Women. Neurourology and Urodynamics, 32, 43-47. https://doi.org/10.1002/nau.22272

[37] Szabo, S.M., Gooch, K.L., Walker, D.R., Johnston, K.M. and Wagg, A.S. (2018) The Association between Overactive Bladder and Falls and Fractures: A Systematic Review. Advances in Therapy, 35, 1831-1841. https://doi.org/10.1007/s12325-018-0796-8 